\title{
LYAPUNOV-TYPE INEQUALITY FOR THIRD-ORDER HALF-LINEAR DIFFERENTIAL EQUATIONS
}

\author{
JOZEF KISEL'ÁK
}

\begin{abstract}
In this paper, we give a proof of a Lyapunov-type inequality for third-order halflinear differential equations. Then some applications, e.g. the distance between consecutive zeros of a solution, are studied with the help of the inequality.
\end{abstract}

\section{Introduction}

In this paper, we generalize the Lyapunov inequality for linear third-order differential equation

$$
y^{\prime \prime \prime}+p(t) y=0
$$

for a class of half-linear differential equations of the third-order. It is well known [6] that if $p \in C[a, b]$ and $x(t)$ is nonzero solution of (1) s.t. $x(a)=x(b)=0(a<b)$ and there exist $d \in[a, b]$ s.t. $y^{\prime \prime}(d)=0$, then the following inequality holds:

$$
\int_{a}^{b}|p(t)| \mathrm{d} s>\frac{4}{(b-a)^{2}}
$$

Such result has found many practical uses in problems as oscillation theory or eigenvalue problems (spectral properties of differential equations). There are several generalizations in the literature. For higher-order linear differential equations see e.g. [1, 8] and for certain nonlinear higher-order differential equations see [5]. Development of theory of differential equations together with practical problems bring also delayed type of equations. If one is interested along this line see [7], where authors handle with the third-order delay differential equations. We study here a special type of nonlinear differential equations, of which the solution space possesses homogeneity property but lacks for additivity property. Second-order half-linear differential equations have been widely studied in recent years and there is a nice

Received June 16, 2011, accepted January 11, 2013.

Communicated by Chung-Tsun Shieh.

2010 Mathematics Subject Classification. 34C10.

Key words and phrases. Lyapunov-type inequality, half-linear, third-order differential equations, oscillatory solution. 
overview in the monograph [2]. Less literature exists, which deals with such equations of higher-order (especially odd-order differential equations), but one can see for example [4].

\section{Main results}

We are concerned with the third-order half-linear differential equation

$$
\left(\frac{1}{r_{2}(t)} \Phi_{\alpha_{2}}\left[\left(\frac{1}{r_{1}(t)} \Phi_{\alpha_{1}}\left[y^{\prime}\right]\right)^{\prime}\right]\right)^{\prime}+q(t) \Phi_{\beta}[y]=0,
$$

where $\alpha_{1}>0, \alpha_{2}>0, \quad q \in C([a, b], \mathbb{R})$, and $\Phi_{\alpha}[x]:=|x|^{\alpha-1} x, \alpha>0$, known as signed-power function. Moreover we assume that $\left(r_{k}\right)^{-1} \in C^{3-k}([a, b],(0, \infty)), k=1,2$, and to preserve mentioned homogeneity property we also demand that $\beta=\alpha_{1} \alpha_{2}$. Equation $(E)$ can be written by means of quasi-derivatives with respect to the coefficients $r_{i}$ and functions $\Phi_{\alpha_{i}}, i=1,2$. We will denote them as follows:

$$
\begin{aligned}
& \mathrm{D}^{(0)} y(t)=y(t), \\
& \mathrm{D}^{(i)} y(t)=\frac{1}{r_{i}(t)} \Phi_{\alpha_{i}}\left[\frac{\mathrm{d}}{\mathrm{d} t} \mathrm{D}^{(i-1)} y(t)\right] \quad i=1,2 \\
& \mathrm{D}^{(3)} y(t)=\frac{\mathrm{d}}{\mathrm{d} t} \mathrm{D}^{(2)} y(t) .
\end{aligned}
$$

A solution of $(E)$ is said to be oscillatory (nonoscillatory) if it has (has not) a sequence of zeros converging to infinity. Equation $(E)$ is oscillatory if all its solutions are oscillatory and nonoscillatory otherwise. If a solution of $(E)$ has two consecutive zeros $a<b$, then there can two cases occur. Either there exist a $d \in[a, b]$ s.t. $\frac{\mathrm{d}}{\mathrm{d} t} \mathrm{D}^{(1)} y(d)=0$ or $\frac{\mathrm{d}}{\mathrm{d} t} \mathrm{D}^{(1)} y(t) \neq 0$ for $t \in[a, b]$. The first case illustrates the following assertion.

Theorem 2.1. If $y(t)$ is a nonzero solution of $(E)$ satisfying $y(a)=y(b)=0$ and there exist $a$ $d \in[a, b]$ s.t. $\frac{\mathrm{d}}{\mathrm{d} t} \mathrm{D}^{(1)} y(d)=0$. Then

$$
2\left(\int_{a}^{b}|q(t)| \mathrm{d} t\right)^{\frac{1}{\beta}}>\min _{c \in[a, b]} h(c)
$$

where $h(c)=\frac{1}{\int_{a}^{c} r_{1}^{\frac{1}{\alpha_{1}}} \mathrm{~d} t\left(\int_{a}^{c} r_{2}(t)^{\frac{1}{\alpha_{2}}} \mathrm{~d} t\right)^{\frac{1}{\alpha_{1}}}}+\frac{1}{\int_{c}^{b} r_{1}^{\frac{1}{\alpha_{1}}} \mathrm{~d} t\left(\int_{c}^{b} r_{2}(t)^{\frac{1}{\alpha_{2}}} \mathrm{~d} t\right)^{\frac{1}{\alpha_{1}}}}$

Proof. We first define functions $y_{k}, k=0,1,2$, as follows:

$$
\begin{array}{ll}
y_{0}:=\mathrm{D}^{(0)} y, & \\
y_{i}:=\mathrm{D}^{(i)} y(t)=\frac{1}{r_{i}(t)} \Phi_{\alpha_{i}}\left[\frac{\mathrm{d}}{\mathrm{d} t} y_{i-1}\right], & i=1,2
\end{array}
$$


Equation $(E)$ is then equivalent to the following differential system:

$$
\begin{aligned}
& y_{i}^{\prime}=r_{i+1}(t)^{\frac{1}{\alpha_{i+1}}} \Phi_{\alpha_{i+1}}^{-1}\left[y_{i+1}\right], \\
& y_{2}^{\prime}=-q(t) \Phi_{\beta}\left[y_{0}\right] .
\end{aligned}
$$

Condition $y_{0}(a)=y_{0}(b)=0$ gives us existence of $c \in(a, b)$ s.t. $y_{0}^{\prime}(c)=0$ and $\left|y_{0}(c)\right|=\max _{t \in[a, b]}\left|y_{0}(t)\right|$. It follows from the latter that $y_{1}(c)=0$. By integrating the first equation of the system (7) from $a$ to $c$ we obtain

$$
y_{0}(c)=\int_{a}^{c} r_{1}^{\frac{1}{\alpha_{1}}}(t) \Phi_{\alpha_{1}}^{-1}\left[y_{1}(t)\right] \mathrm{d} t
$$

which implies

$$
\left|y_{0}(c)\right| \leq \int_{a}^{c} r_{1}^{\frac{1}{\alpha_{1}}}(t)\left|y_{1}(t)\right|^{\frac{1}{\alpha_{1}}} \mathrm{~d} t .
$$

Now let $t$ be in $[a, c]$. From the fact that $y_{1}(c)=0$ and $y_{1}(t)=\int_{c}^{t} y_{1}^{\prime}(s) \mathrm{d} s$ we get

$$
\left|y_{1}(t)\right| \leq \int_{a}^{c} r_{2}^{\frac{1}{\alpha_{2}}}(t)\left|y_{2}(t)\right|^{\frac{1}{\alpha_{2}}} \mathrm{~d} t
$$

Further, from the second condition of the assumptions and relations (6) or (7), we know that $y_{2}(d)=0$, which implies $y_{2}(t)=-\int_{d}^{t} q(s) \Phi_{\beta}\left[y_{0}(s)\right] \mathrm{d} s$ for $t \in[a, c]$. Moreover we have

$$
\left|y_{2}(t)\right|<\left|y_{0}(c)\right|^{\beta} \int_{a}^{b}|q(t)| \mathrm{d} t .
$$

Combining inequalities (8)-(10), we get

$$
\left|y_{0}(c)\right|<\left|y_{0}(c)\right| \int_{a}^{c} r_{1}^{\frac{1}{\alpha_{1}}}(t) \mathrm{d} t\left(\int_{a}^{c} r_{2}^{\frac{1}{\alpha_{2}}}(t) \mathrm{d} t\right)^{\frac{1}{\alpha_{1}}}\left(\int_{a}^{b}|q(t)| \mathrm{d} t\right)^{\frac{1}{\beta}},
$$

which implies

$$
1<\int_{a}^{c} r_{1}^{\frac{1}{\alpha_{1}}}(t) \mathrm{d} t\left(\int_{a}^{c} r_{2}^{\frac{1}{\alpha_{2}}}(t) \mathrm{d} t\right)^{\frac{1}{\alpha_{1}}}\left(\int_{a}^{b}|q(t)| \mathrm{d} t\right)^{\frac{1}{\beta}} .
$$

Analogously, we can get

$$
1<\int_{c}^{b} r_{1}^{\frac{1}{\alpha_{1}}}(t) \mathrm{d} t\left(\int_{c}^{b} r_{2}^{\frac{1}{\alpha_{2}}}(t) \mathrm{d} t\right)^{\frac{1}{\alpha_{1}}}\left(\int_{a}^{b}|q(t)| \mathrm{d} t\right)^{\frac{1}{\beta}}
$$

But (11) and (12) together imply (5). Moreover, it is obvious that $h$ takes its minimum in $(a, b)$, since it is continuous there and $\lim _{c \rightarrow a^{+}} h(c)=\lim _{c \rightarrow b^{-}} h(c)=\infty$.

In the case that $\frac{\mathrm{d}}{\mathrm{d} t} \mathrm{D}^{(1)} y(t) \neq 0$ for $t \in[a, b]$, we consider three consecutive zeros of $y(t)$. We give only sketch of the proof as it is almost copy of the previous one. 
Theorem 2.2. If $y(t)$ is a nonzero solution of $(E)$ satisfying $y(a)=y(d)=y(b)=0, \frac{\mathrm{d}}{\mathrm{d} t} \mathrm{D}^{(1)} y(t) \neq$ 0 for $t \in[a, d]$ and $y(t) \neq 0$ for $t \in(a, d) \cup(d, b)$. Then inequality (5) holds.

Proof. Conditions $y_{0}(a)=y_{0}(d)=y_{0}(b)=0$ give us existence of $c_{1} \in(a, d), c_{2} \in(d$, $b)$, s.t. $y_{1}\left(c_{1}\right)=y_{1}\left(c_{2}\right)=0$ and further application of Rolle's theorem gives us existence of $e \in\left(c_{1}, c_{2}\right)$, s.t. $y_{2}(e)=0$. Denoting by $c \in(a, d) \cup(d, b)$ a point where $\max _{t \in[a, b]}\left|y_{0}(t)\right|=\left|y_{0}(c)\right|$ and using previous procedure it can be proved that inequality (5) holds. Notice, that there can not be problem with continuity of $h$ on $(a, b)$.

Remark 2.1. Put $r_{1}(t)=r_{2}(t)=1$. Since $h$ attains minimum at $c=\frac{a+b}{2}$, then (5) reduces to

$$
\int_{a}^{b}|q(t)| \mathrm{d} t>\left(\frac{2}{b-a}\right)^{\alpha_{2}\left(\alpha_{1}+1\right)} .
$$

Notice that in case $\alpha_{1}=\alpha_{2}=1$ this inequality reduces to (2), which appears in the classical result.

Remark 2.2. Put $r_{1}(t)=r_{2}(t)=r(t)$ and $\alpha_{1}=\alpha_{2}=\alpha$ then (5) reduces to

$$
\int_{a}^{b}|q(t)| \mathrm{d} t>\left(\frac{2}{\int_{a}^{b} r(t)^{\frac{1}{\alpha}} \mathrm{d} t}\right)^{\alpha(\alpha+1)},
$$

since $\int_{a}^{c} r(t)^{\frac{1}{\alpha}} \mathrm{d} t=\int_{c}^{b} r(t)^{\frac{1}{\alpha}} \mathrm{d} t=\frac{1}{2} \int_{a}^{b} r(t)^{\frac{1}{\alpha}} \mathrm{d} t$ must hold for $c$ in order to minimize $h$ on $(a, b)$ $\left(c=R^{-1}\left(\frac{R(b)+R(a)}{2}\right)\right.$, where $R$ is antiderivative of $\left.r^{\frac{1}{\alpha}}\right)$.

\section{Applications}

Further we introduce some applications of the previous results for reduced equation $(E)$.

Theorem 3.1. Let $y(t)$ be an oscillatory solution of the reduced $\left(r_{1}(t)=r_{2}(t)=1\right)$ equation (E) with increasing sequence of zeros $\left\{t_{k}\right\}_{k=1}^{\infty}$ and $q \in L^{\mu}([0, \infty], \mathbb{R}), \mu \in[1, \infty)$. Then distances between consecutive zeros $\left\{t_{k+1}-t_{k}\right\}$ or $\left\{t_{k+2}-t_{k}\right\}$ goes to infinity.

Proof. In a proof by contradiction we suppose that, in the case that $\frac{\mathrm{d}}{\mathrm{d} t} \mathrm{D}^{(1)} y(t)=0$ for some $t \in\left[t_{k}, t_{k+1}\right]$ for every large $k$, is not true that $\left\{t_{k+1}-t_{k}\right\} \rightarrow \infty$. Hence, there exist a subsequence $\left\{t_{k_{n}}\right\}_{n=1}^{\infty}$ s.t. $\left(t_{k_{n+1}}-t_{k_{n}}\right)<K$ for every $n,(K>0)$. Let $\frac{\mathrm{d}}{\mathrm{d} t} \mathrm{D}^{(1)} y\left(c_{k_{n}}\right)=0$ for $c_{k_{n}} \in\left[t_{k_{n}}, t_{k_{n+1}}\right]$ and $\max _{t \in\left[t_{k_{n}}, t_{k_{n+1}}\right]}|y(t)|=\left|y\left(d_{k_{n}}\right)\right|$ where $d_{k_{n}} \in\left(t_{k_{n}}, t_{k_{n+1}}\right)$. Without loss of generality we can assume that $c_{k_{n}}<d_{k_{n}}$. Then it follows that

$$
\int_{t_{k_{n}}}^{t_{k_{n+1}}}|q(t)| \mathrm{d} t>\left(\frac{2}{d_{k_{n}}-t_{k_{n}}}\right)^{\beta+\alpha_{2}} .
$$


From integrability of $q$ we have

$$
\int_{t_{k_{n}}}^{\infty}|q(t)|^{\mu} \mathrm{d} t<\left(\frac{2^{\beta+\alpha_{2}}}{K^{\beta+\alpha_{2}+\frac{1}{\mu}}}\right)^{\mu}
$$

for sufficiently large $n$ and $\frac{1}{\mu}+\frac{1}{\tilde{\mu}}=1$. Therefore using Hölder inequality we obtain

$$
\begin{gathered}
\left.1<\left(\frac{d_{k_{n}}-t_{k_{n}}}{2}\right)^{\beta+\alpha_{2}} \int_{t_{k_{n}}}^{t_{k_{n+1}}}|q(t)| \mathrm{d} t \leq \frac{\left(t_{k_{n+1}}-t_{k_{n}}\right)^{\beta+\alpha_{2}+\frac{1}{\mu}}}{2^{\beta+\alpha_{2}}}\left(\int_{t_{k_{n}}}^{t_{k_{n+1}}}|q(t)|^{\mu} \mathrm{d} t\right)\right)^{\frac{1}{\mu}} \leq \\
\left.\leq \frac{\left(t_{k_{n+1}}-t_{k_{n}}\right)^{\beta+\alpha_{2}+\frac{1}{\mu}}}{2^{\beta+\alpha_{2}}}\left(\int_{t_{k_{n}}}^{\infty}|q(t)|^{\mu} \mathrm{d} t\right)\right)^{\frac{1}{\mu}}<\frac{K^{\beta+\alpha_{2}+\frac{1}{\mu}}}{2^{\beta+\alpha_{2}}}\left(\frac{2^{\beta+\alpha_{2}}}{K^{\beta+\alpha_{2}+\frac{1}{\mu}}}\right)=1,
\end{gathered}
$$

a contradiction.

Now suppose that $\frac{\mathrm{d}}{\mathrm{d} t} \mathrm{D}^{(1)} y(t) \neq 0$ for $t \in\left[t_{k}, t_{k+1}\right]$ (for some large $k$ ). In this case we consider three consecutive zeros $t_{k}<t_{k+1}<t_{k+2}$. Suppose that there exist subsequence $\left\{t_{k_{n}}\right\}_{n=1}^{\infty}$ s.t. $\left(t_{k_{n+1}}-t_{k_{n}}\right)<M$ for every $n,(M>0)$ and $\frac{\mathrm{d}}{\mathrm{d} t} \mathrm{D}^{(1)} y(t) \neq 0$ for $t \in\left[t_{k_{n}}, t_{k_{n+1}}\right]$. Since $y\left(t_{k_{n+2}}\right)=0$, there exists a $c_{k_{n}} \in\left[t_{k_{n+1}}, t_{k_{n+2}}\right]$ such that $\frac{\mathrm{d}}{\mathrm{d} t} \mathrm{D}^{(1)} y\left(c_{k_{n}}\right)=0$. Now set $\max _{t \in\left[t_{k_{n}}, t_{k_{n+2}}\right]}|y(t)|=\left|y\left(d_{k_{n}}\right)\right|$ where $d_{k_{n}} \in\left(t_{k_{n}}, t_{k_{n+1}}\right) \cup\left(t_{k_{n+1}}, t_{k_{n+2}}\right)$. If $d_{k_{n}} \in\left(t_{k_{n+1}}, t_{k_{n+2}}\right)$ then we can proceed as in the previous part of the proof. If $d_{k_{n}} \in\left(t_{k_{n}}, t_{k_{n+1}}\right)$, then it follows that

$$
\int_{t_{k_{n}}}^{t_{k_{n+2}}}|q(t)| \mathrm{d} t>\left(\frac{2}{d_{k_{n}}-t_{k_{n}}}\right)^{\beta+\alpha_{2}}
$$

Therefore using Hölder inequality we obtain a contradiction as in the first part of the proof.

The following theorems give us an estimation (upper bound) of the number of zeros of an oscillatory solution of reduced equation $(E)$ on bounded interval $[0, T]$.

Theorem 3.2. If $y(t)$ is an oscillatory solution of reduced $\left(r_{1}(t)=r_{2}(t)=1\right)$ equation (E) with zeros $0<t_{1}<t_{2}<\cdots<t_{N} \leq T$ and $\frac{\mathrm{d}}{\mathrm{d} t} \mathrm{D}^{(1)} y\left(e_{k}\right)=0$ for some $e_{k} \in\left[t_{k}, t_{k+1}\right], k=1,2, \ldots, N-1$. Moreover let $\beta+\alpha_{2} \geq 1$, then

$$
T^{\beta+\alpha_{2}} \int_{0}^{T}|q(t)| \mathrm{d} t>2^{\beta+\alpha_{2}}(N-1)^{\beta+\alpha_{2}+1} .
$$

Proof. We know that $\int_{t_{k}}^{t_{k+1}}|q(t)| \mathrm{d} t>\left(\frac{2}{t_{k+1}-t_{k}}\right)^{\beta+\alpha_{2}}, k=1, \ldots, N-1$. Thus,

$$
\int_{0}^{T}|q(t)| \mathrm{d} t \geq \int_{t_{1}}^{t_{N}}|q(t)| \mathrm{d} t>\sum_{k=1}^{N-1}\left(\frac{2}{t_{k+1}-t_{k}}\right)^{\beta+\alpha_{2}} .
$$


Now, using known inequality for the power mean with exponent $\beta+\alpha_{2}$ and arithmetic mean and inequality $\frac{1}{n} \sum_{i=1}^{n} A_{i} \geq\left(\prod_{i=1}^{n} A_{i}\right)^{\frac{1}{n}} \geq\left(\frac{1}{n} \sum_{i=1}^{n} \frac{1}{A_{i}}\right)^{-1}, A_{i}>0, \quad 1 \leq i \leq n$, we obtain

$$
\begin{aligned}
\sum_{k=1}^{N-1}\left(\frac{1}{t_{k+1}-t_{k}}\right)^{\beta+\alpha_{2}} & \geq(N-1)\left(\frac{1}{N-1} \sum_{k=1}^{N-1} \frac{1}{t_{k+1}-t_{k}}\right)^{\beta+\alpha_{2}} \geq(N-1)^{\beta+\alpha_{2}+1}\left(\sum_{k=1}^{N-1}\left(t_{k+1}-t_{k}\right)\right)^{-\beta-\alpha_{2}} \\
& =(N-1)^{\beta+\alpha_{2}+1}\left(t_{N}-t_{1}\right)^{-\beta-\alpha_{2}}>\frac{(N-1)^{\beta+\alpha_{2}+1}}{T^{\beta+\alpha_{2}}} .
\end{aligned}
$$

This completes the proof.

Theorem 3.3. If $y(t)$ is an oscillatory solution of reduced $\left(r_{1}(t)=r_{2}(t)=1\right)$ equation $(E)$ with zeros $0<t_{1}<t_{2}<\cdots<t_{2 N+1} \leq T$ and $\frac{\mathrm{d}}{\mathrm{d} t} \mathrm{D}^{(1)} y(t) \neq 0$ for $t \in\left[t_{2 k-1}, t_{2 k}\right], k=1,2, \ldots, N$. Moreover let $\beta+\alpha_{2} \geq 1$, then

$$
T^{\beta+\alpha_{2}} \int_{0}^{T}|q(t)| \mathrm{d} t>2^{\beta+\alpha_{2}} N^{\beta+\alpha_{2}+1}
$$

The proof can be omitted as one can proceed similarly as in Theorem 3.2. We left the case $\beta+\alpha_{2} \in(0,1)$ as an open problem.

Example 3.1. For simplicity we consider exponents $\alpha_{1}, \alpha_{2}$ to be the quotients of two odd numbers. Moreover, let the coefficients of the quasi-derivatives be identically constant. We study the following generalized Euler's differential equation on $\mathbb{R}^{+}$

$$
\left(\left[\left(\left[y^{\prime}\right]^{\alpha_{1}}\right)^{\prime}\right]^{\alpha_{2}}\right)^{\prime}+\frac{\gamma}{(t+1)^{\beta+\alpha_{2}+1}} y^{\beta}=0 .
$$

We can proceed using the analogy with the linear Euler differential equation. If we denote as $D=\left(\alpha_{1}+\alpha_{2}\right)^{2}+4 \beta\left(\beta+\alpha_{2}\right)$, then the roots of an algebraic (indical) equation corresponding to a solution $t^{\lambda}$ are

$$
\lambda_{ \pm}=\frac{\alpha_{1}+2 \beta\left(1+\alpha_{1}\right)+\alpha_{2} \pm \sqrt{D}}{2 \alpha_{1}\left(1+\beta+\alpha_{2}\right)} .
$$

Although it has not been proven yet and it is only a conjecture, see [3], we believe that it can be shown the following. Constants $\gamma_{ \pm}=\lambda_{ \pm}^{\beta}\left(\lambda_{ \pm}-1\right)^{\alpha_{2}} \alpha_{1}^{\alpha_{2}}\left(\beta\left(\lambda_{ \pm}-1\right)-\alpha_{2}\right)$ decide whether (13) is oscillatory or not (notice, that in the linear case $\gamma_{ \pm}=2 \sqrt{3} / 9$ ). Be more precise, a conjecture states that if $\gamma \in\left[\gamma_{-}, \gamma_{+}\right]$then equation (13) is nonoscillatory, otherwise it is oscillatory. Thus we can state at least estimate for $\gamma$. So, from the previous reflections we have

$$
\frac{|\gamma|\left(1-(T+1)^{-\beta-\alpha_{2}}\right) T^{\beta+\alpha_{2}}}{\beta+\alpha_{2}}>2^{\beta+\alpha_{2}}(N-1)^{\beta+\alpha_{2}+1} .
$$


Example 3.2. Finally we give an application of the obtained result for the following eigenvalue problem

$$
\begin{aligned}
& \mathrm{D}^{(3)} y \pm \lambda q(t) \Phi_{\beta}[y]=0, \\
& y(a)=y(c)=y(b)=0, \quad a<c<b .
\end{aligned}
$$

Let the assumptions of Theorem 2.1 be fulfilled, then it follows that

$$
|\lambda|>\frac{H^{\beta}}{2^{\beta} \int_{a}^{b}|q(t)| \mathrm{d} t},
$$

where $H=\min _{c \in[a, b]} h(c)$ and $h$ is function defined in Theorem 2.1. Especially for the reduced problem $\left(r_{1}(t)=r_{2}(t)=1\right)$ we obtain

$$
|\lambda|>\frac{2^{\beta+\alpha_{2}}}{(b-a)^{\beta+\alpha_{2}} \int_{a}^{b}|q(t)| \mathrm{d} t} .
$$

\section{References}

[1] D. Çakmak, Lyapunov-type integral inequalities for certain higher order differential equations, Appl. Math. Comput., 216(2010), 368-373.

[2] O. Došlý and P. Řehák, Half-linear differential equations, North-Holland Mathematics Studies 202. Amsterdam: Elsevier. xiv, 517 p., 2005.

[3] J. Kisel'ák, Integral comparison theorem for half-linear third-order differential equations, Adv. Differ. Equ. Control Process, 8(2011), 23-32.

[4] M. Naito, Existence and asymptotic behavior of positive solutions of higher-order quasilinear ordinary differential equations, Math. Nachr., 279(2006), 198-216.

[5] S. Panigrahi, Liapunov-type integral inequalities for certain higher-order differential equations, Electron. J. Differ. Equ., 2009(2009), 1-14.

[6] S. Panigrahi and N. Parhi, On Liapunov-type inequality for third-order differential equations, J. Math. Anal. Appl., 233(1999), 445-460.

[7] N. Parhi, and S. Panigrahi, Lyapunov-type inequality for delay-differential equations of third order, Czech. Math. J., 52(2002), 385-399.

[8] X. Yang, Y.-I. Kim and K. Lo, Lyapunov-type inequality for a class of odd-order differential equations, J. Comput. Appl. Math., 234(2010), 2962-2968.

Institute of Mathematics, Faculty of Science, P.J.Šafárik University in Košice, Jesenná 5, 04001 Košice, Slovakia.

E-mail: jozef.kiselak@upjs.sk 